Results Mean delta weight Z-score increased from -1.8 to -1.08 (figure 4) following the implementation of all of the noted interventions with centerline shift that has lasted for 8 months (40\% improvement). Mean number of lactation consults ordered per week increased from 1 to 4 .

Conclusions Reducing nutrition practice variation shows marked improvements in infant nutrition status. We continue to test other interventions with the hope of further decreasing growth failure.

\section{IHI ID 07 DECREASING EMERGENCY DEPARTMENT LENGTH OF STAY FOR ADMITTED PEDIATRIC PATIENTS}

${ }^{1}$ Lindsay Carter, ${ }^{1}$ Kristen Solemina, ${ }^{1}$ Jessica Mascola, ${ }^{2}$ Michael Peer, ${ }^{1}$ Peter Greenspan. ${ }^{1}$ MassGeneral Hospital for Children, USA; ${ }^{2}$ Massachusetts General Hospital, USA

\subsection{6/ihisciabs.7}

Background Prolonged emergency department (ED) length of stay (LOS) can lead to safety risks including handovers, medication and meal delays, high patient to nursing ratios, and lack of accommodations for families, potentially negatively affecting patient care and satisfaction. In 2016, 75\% of admitted pediatric patients at MassGeneral Hospital for Children spent $>2$ hours waiting for a bed.

Objectives To decrease ED median LOS of admitted pediatric patients by $8 \%$ in 12 months.

Methods Four working groups targeted the process and developed interventions. Primary outcome measure was ED LOS. Process measures included provider and nursing handoffs within $30 \mathrm{~min}$ of an available inpatient bed and percentage of inpatient pre-noon discharges. Balance measures included safety report filings regarding ED transfers and readmissions within 48 hours of discharge. Baseline median and variability was determined through statistical process control charts using healthcare rules of interpretation for improvement analysis.

Results There were 12 months of baseline and 16 months of intervention involving 4846 patients. Median LOS decreased by $10.1 \%$, from 5.73 hours to 5.15 hours. MD and RN handoffs within $30 \mathrm{~min}$ of bed availability improved by $11.4 \%$ and $17.9 \%$, respectively. There was no statistically significant change in percentage of pre-noon discharges. There was no increase in safety reports or readmissions.

Conclusions Through a multimodal intervention process involving education, process measures, and engaged leadership, there was a significant improvement in ED LOS for admitted pediatric patients without increases in readmissions or safety issues. Initiatives are ongoing to address barriers to timely inpatient discharges and sustaining the intervention over time.

\section{IHI ID 08 EMERGENCY MEDICAL SERVICES TREATMENT DISPARITIES BY PATIENT RACE}

Jamie Kennel. Oregon Health and Science University/Oregon Tech, US

\subsection{6/ihisciabs.8}

Background In the US racial minorities are at a greater risk of receiving a lower quality of medical care. While hundreds of documented studies have identified racial treatment disparities in many areas of medicine, the field of Emergency Medical Services (EMS) remains relatively unexamined.

Objectives This study explores racial treatment disparities in EMS pain management practices for adult patients who engage with pre-hospital emergency medical services for traumatic or painful emergencies.

Methods This study is a quantitative analysis of 104210 medical charts from 63 EMS agencies in Oregon from 2015 through 2017. Primary measures include the receipt of pain medication (outcome), patient race (predictor), and numerous control variables including EMS provider impression, pain severity, and socioeconomic status (SES) covariates.

Results African American patients were 40\% less likely (adjusted OR 0.60, 95\% CI 0.53 to 0.68 ), and Asian patients were $36 \%$ less likely (adjusted OR 0.64 , 95\% CI 0.50 to 0.83 ), to receive any pain medication compared to White patients while controlling for common clinical and SES confounders. Secondary analysis indicated African Americans with private insurance experienced an even larger disparity (adjusted OR 0.45 , 95\% CI 0.31 to 0.64 ) compared to White patients with private insurance.

Conclusions African American patients and Asian patients in Oregon receiving pre-hospital emergency medical assistance for painful injuries or conditions are significantly less likely to receive the same treatment as White patients. While this finding is consistent with many studies in the Emergency Department, these results demonstrate that racial disparities exist in EMS treatment.

\section{IHI ID 09 A QUALITY IMPROVEMENT (QI) INITIATIVE TO DECREASE DIAGNOSTIC ERRORS}

Richard McClead, Rena Kasick, Michael Perry, Jennifer Melvin, Kelly Kersey, Manmohan Kamboj, Ivor Hill, Garey Noritz, Ryan Bode, Jack Stevens, Taylor Ballenger, Jeff Hoffman. Nationwide Children's Hospital, USA

\subsection{6/ihisciabs.9}

Background Diagnostic errors (DE) in healthcare are a widespread, but underappreciated, problem. Investigators report that everyone will experience at least one DE in their lifetime.

Objectives After the occurrence of six serious adverse events related to DE in the first six months of 2015, we chartered a QI team in 2016 to decrease DE.

Methods The team used QI methodology, established a specific aim and key driver diagram (figure 1), and developed the diagnostic error index (DEI 1.0) to measure the impact of interventions to decrease DE. The DEI 1.0 consists of 5 sources of DE:

1. class I autopsy findings,

2. RCA with DE as a failure mode,

3. medical record triggers,

4. Morbidity \& Mortality reports, and

5. other adverse event reports of DE.

We aimed to reduce the DEI from $7 /$ month to $3 /$ month by December 31, 2018. We evaluated several interventions to decrease DE including the diagnostic time out, open notes, differential diagnosis (DDx) software, EMR advisory board, pan-ophthalmoscopy, admission DDx audits, and participation in a pilot SIDM-IHI DE collaborative. 